\title{
Alterations in Adult Rat Heart after Neonatal Dexamethasone Therapy
}

\author{
WILLEM B. DE VRIES, FEIKE R. VAN DER LEIJ, JOOST M. BAKKER, \\ PATRICK J.G.H. KAMPHUIS, MATTHIJS F.M. VAN OOSTERHOUT, \\ MARGUERITE E.I. SCHIPPER, GIOIA B. SMID, BEATRIJS BARTELDS, AND FRANK VAN BEL \\ Departments of Neonatology [W.B.d.V., J.M.B., F.v.B.], Pediatric Immunology [J.M.B.], and Pathology \\ [M.F.M.v.O., M.E.I.S.], and the Rudolf Magnus Institute for Neurosciences [P.J.G.H.K.], University \\ Medical Center/Wilhelmina Children's Hospital, Utrecht, The Netherlands, and Department of Pediatrics \\ [F.R.v.d.L., G.B.S., B.B.], Groningen University Institute for Drug Exploration/Beatrix Children's \\ Hospital, Groningen, The Netherlands
}

\section{ABSTRACT}

\begin{abstract}
Glucocorticoid treatment in preterm babies to prevent chronic lung disease causes myocardial hypertrophy and increased myocardial protein content. Although these changes are thought to be transient, there is evidence that dexamethasone (DEX) induces permanent myocardial abnormalities as well. We investigated whether a therapeutic course of neonatal DEX in rat pups produces anatomic and biochemical alterations in rat hearts during adult life. Twenty-four rat pups were treated with DEX on $\mathrm{d} 1,2$, and $3(0.5,0.3$, and $0.1 \mu \mathrm{g} / \mathrm{g})$ of life, with doses proportional to those used in preterm babies. Twenty-four control pups were treated with saline. At d 7, wk 8 , or wk $45(n=8$ per group) rats were killed. The anatomic parameters measured were body weight ( $\mathrm{Bw}$, in grams), heart (myocardial) weight (Hw, in milligrams), and the $\mathrm{Hw}: \mathrm{Bw}$ ratio. Myocardial total protein (Prot) and DNA content were determined, and the Prot:DNA ratio was calculated. Histopathology and morphometry were performed on 45-wk-old rat hearts. In DEX-treated rat pups, at d 7, Bw and Hw were lower and the Hw:Bw ratio was increased. DNA content was lower, Prot higher, and Prot:DNA ratio was increased. In 8-wk-old rats Bw, Hw, DNA content, Prot content
\end{abstract}

or Prot:DNA ratio did not differ between groups, but the Prot:DNA ratio still tended to be higher in DEX-treated rats. In 45-wk-old rats $\mathrm{Hw}$ and $\mathrm{Hw}$ :Bw ratio were significantly lower and Prot:DNA ratio higher in DEX-treated rats. Histopathologic analysis showed larger cardiomyocyte volume, length, and width, indicating hypertrophy, and increased collagen, indicating early degeneration of individual myocytes. In conclusion, neonatal DEX treatment in rat pups causes a permanent decrease in heart weight, as well as hypertrophy and early degeneration of cardiomyocytes during adulthood. (Pediatr Res 52: 900-906, 2002)
Bw, body weight
DEX, dexamethasone
GC, glucocorticoid
Hw, heart weight
Prot, total protein
SAL, saline

Abbreviations
Chronic lung disease is a common outcome in extremely preterm neonates with severe respiratory distress syndrome and carries a high incidence of morbidity and mortality. Inflammation is an important factor in its pathogenesis. Because of their antiinflammatory properties GCs, particularly DEX, resulted in an impressive decrease in chronic lung disease $(1,2)$. Consequently GCs are frequently used and have become indispensable in all neonatal intensive care units in the Western world,

Received August 21, 2001; accepted June 6, 2002.

Correspondence: Frank van Bel, M.D., Ph.D., Wilhelmina Children's Hospital, Room KE 04.123.1, Department of Neonatology, PO Box 85090, 3508 AB Utrecht, The Netherlands; e-mail: F.vanBel@wkz.azu.nl

Partly supported by The Netherlands Heart Foundation (FvdL grants NHS 97.093 and NHS 2001.081).

DOI: 10.1203/01.PDR.0000036281.74804.27 especially inasmuch as an alternative therapy is not available at present. Despite the short-term benefits of GCs, major concern has emerged now because recent experimental studies report long-lasting adverse effects of perinatal GC treatment in later life, such as lifetime changes in cardiovascular function and reprogramming of the neuroendocrine system (3-5). Benediktsson et al. (6) reported that GC exposure during the perinatal period leads to hypertension during adult life, possibly related to a GC-induced reprogramming of the hypothalamic-pituitary-adrenal axis. Neonatal GCs also altered the epinephrine and norepinephrine content in the hypothalamus (7). Experimental and human studies revealed that neonatal GC treatment induced transient hypertrophy of the myocardium with changes in the Prot:DNA ratio and in the actin content of the heart $(8,9)$. Moreover, Rudolph et al. (10) found that 
prenatally administered GCs changed the pattern of myocardial growth in fetal lambs. Therefore we were interested in whether the neonatally GC-induced hypertrophy and changes in growth of the myocardium are reversible or permanent, inducing lifetime anatomic and biochemical myocardial changes leading to cardiovascular disease during adult life.

We investigated anatomic, biochemical, and histopathologic properties of the myocardium of neonatal, juvenile, and adult rats treated with DEX during early neonatal life (in a dose proportional to that given to human preterm babies) as compared with a placebo.

\section{MATERIALS AND METHODS}

Animals. Ten-day-pregnant Wistar rats (250-280 g; Central Animal Laboratory, Utrecht University, Utrecht, The Netherlands) were housed individually. They were kept under conventional conditions (dark phase, 1900-0700 h) with free access to commercial rat food and water. Pups were born on $\mathrm{d}$ 22-23 of gestation. On the day of birth (designated d 0), all pups were removed from the nests, and eight healthy pups (four females and four males) were randomly returned to each dam. Pups were weaned at $21 \mathrm{~d}$ of age and housed two or three per cage. All experimental procedures were approved by the Committee for Animal Experimentation of the University of Utrecht.

Experimental design. Twenty-four rat pups were treated intraperitoneally with DEX 21-phosphate on neonatal d 1, 2, and $3(0.5,0.3$, and $0.1 \mu \mathrm{g} / \mathrm{g})$. The dose and duration of the treatment was based on and proportional to a 21-d tapering course of DEX (starting dose, $0.5 \mathrm{mg} / \mathrm{kg}$ ) to prevent or reduce chronic lung disease in preterm infants: for extrapolation of this human treatment protocol to the neonatal rat, the exposure to DEX can be estimated as a percentage of the total lactation period in humans $(4-6 \mathrm{mo})$. Inasmuch as in rats the lactation period is $21 \mathrm{~d}$, rat pups were therefore treated with the abovementioned tapering course of $3 \mathrm{~d}$. Twenty-four control pups were included and treated with equivalent volumes of SAL. At $7 \mathrm{~d}, 8 \mathrm{wk}$, and $45 \mathrm{wk}$ of life ( $n=8$ per group) rats were killed. The following anatomic measurements were collected: $\mathrm{Bw}$ (in grams), $\mathrm{Hw}$ (myocardial, in milligrams), and the ratio of $\mathrm{Hw}$ to $\mathrm{Bw}$. For this study only female offspring were used in both SAL-treated as well as DEX-treated rat pups.

Total ventricular DNA and Prot measurements. Heart wet weight was determined, and the ventricles were homogenized in saline. Total DNA content was measured by the colorimetric diphenylamine method according to Burton as modified by Knowlton et al. (11). In brief, a volume of $25-100 \mu \mathrm{L}$ of the homogenate was precipitated and washed in $2 \% \mathrm{HClO}_{4}$. Centrifugation was at $1500 \times g$, and the pellet was hydrolyzed for $20 \mathrm{~min}$ at $70^{\circ} \mathrm{C}$ in $2 \% \mathrm{HClO}_{4}$. After cooling down, $1 \mathrm{~mL}$ of diphenylamine solution $(1.5 \mathrm{~g}$ of diphenylamine, $100 \mathrm{~mL}$ of glacial acetic acid, $1.5 \mathrm{~mL}$ of glacial sulfuric acid plus freshly added $5 \mu \mathrm{L} / \mathrm{mL}$ of $1.6 \%$ acetaldehyde) was added, and the reaction was allowed to take place overnight at room temperature. Absorption was measured at $600 \mathrm{~nm}$ in a Hitachi model 100-40 spectrophotometer (Goffin Meyvis, Etten-Leur, The Netherlands). A standard series of 25-250 $\mu \mathrm{g}$ of salmon sperm DNA (Sigma Chemical Co., St. Louis, MO, U.S.A.) was used to calculate absolute amounts of DNA. Protein measurements were by the bicinchoninic acid method (12) as supplied in a kit (Pierce, Rockford, IL, U.S.A.). The actual amounts of DNA and Prot of the combined left and right ventricles were determined, and the Prot:DNA ratio (micrograms of protein to micrograms of DNA) was calculated.

Histopathologic analysis. Morphologic and morphometric analysis was performed on two additional groups of female rats at 45 wk of age. One group consisting of four pups was treated with DEX, one group also consisting of four rat pups was treated with SAL, in a similar manner as indicated in the experimental design. For histopathologic examination the hearts were immersion fixed in phosphate-buffered formalin $4 \%$ and embedded in paraffin. The hearts were sectioned parallel to the equator in 3-mm sections and routinely stained with hematoxylin and eosin for conventional histopathologic analysis. The equatorial section was used to analyze myocyte dimensions and collagen content as described before $(13,14)$. The sections were stained with a modification of the AZAN technique. For each heart the median value of myocyte surface area, length, and width of 30 longitudinally oriented myocytes was determined using a Videoplan interactive analysis system. To control for proper longitudinal orientation, only those myocytes that showed intercalated discs on both sides and a nucleus in the center of the cell were measured. These myocytes were found at the middle third portion of transversally sectioned myocardium. Myocyte volume was calculated as area (in the longitudinal section) multiplied by width times $\pi / 4$ on the assumption of a cylindrical configuration. The same sections were also stained with Sirius Red (Polysciences, Warrington, PA, U.S.A.) to determine the collagen-positive area in 45 fields (magnification $\times 200$ ), excluding epicardial and endocardial as well as perivascular areas. Collagen content was expressed as fraction of the total myocyte area. The sample size for myocyte morphometry and collagen assay was chosen on the basis of a progressive means test, indicating that with the sample sizes

Table 1. Mean values ( \pm SD) of Bw, Hw, Prot, and DNA content of neonatally SAL-and DEX-treated rats at d 7, wk 8, and wk 45 of age

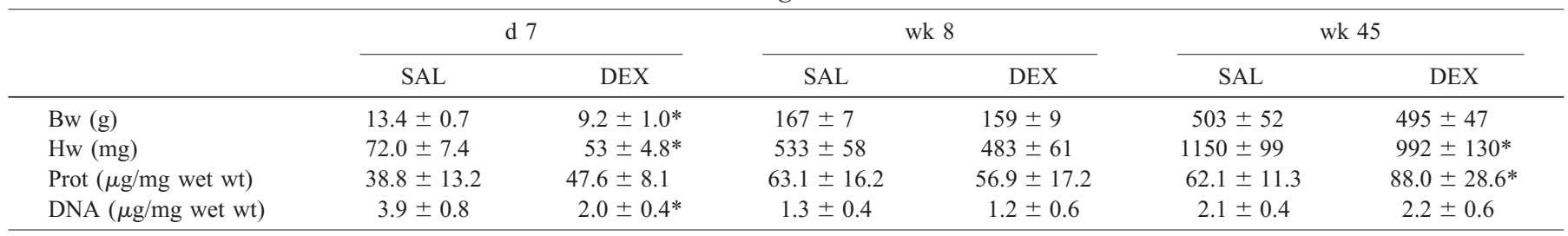

$* p<0.001$ vs SAL-treated rat pups. 


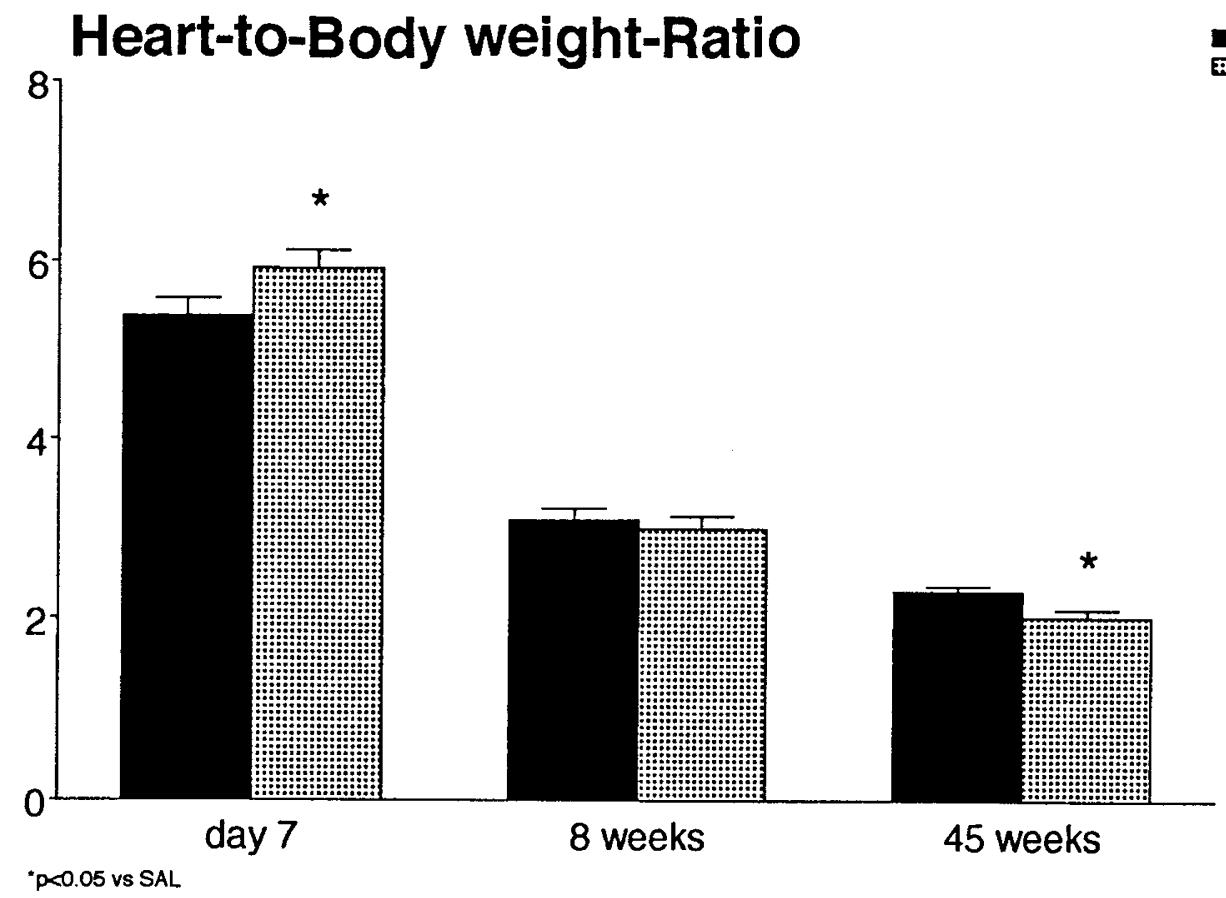

- SAL

円 DEX

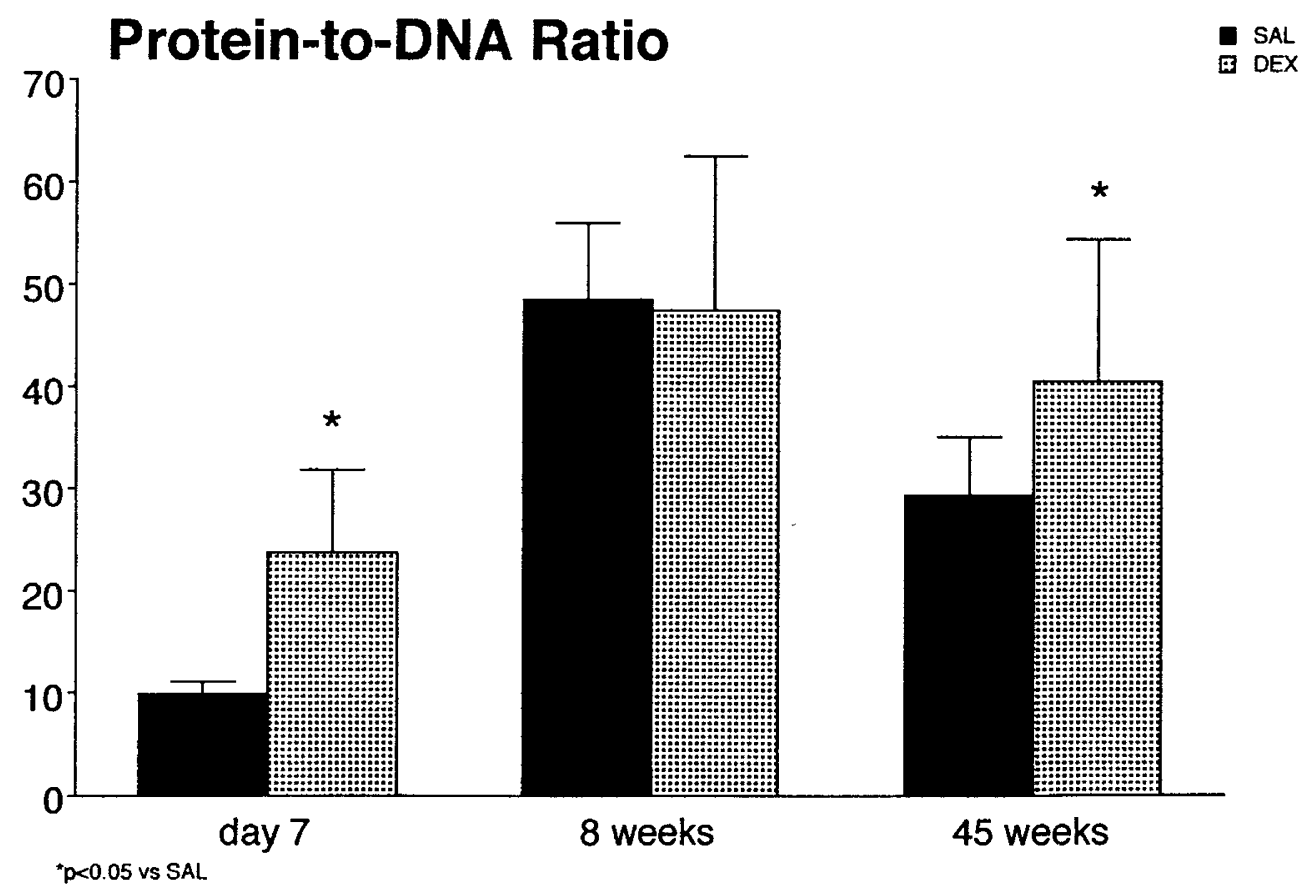

Figure 1. Mean values ( $\pm \mathrm{SD}$ ) of Hw:Bw ratio (top) and of Prot:DNA ratio (bottom) in 7-d-, 8-wk-, and 45-wk-old rats treated neonatally with DEX (strippled bars) or SAL (black bars). ${ }^{*} p<0.05$ DEX $v s$ SAL. 

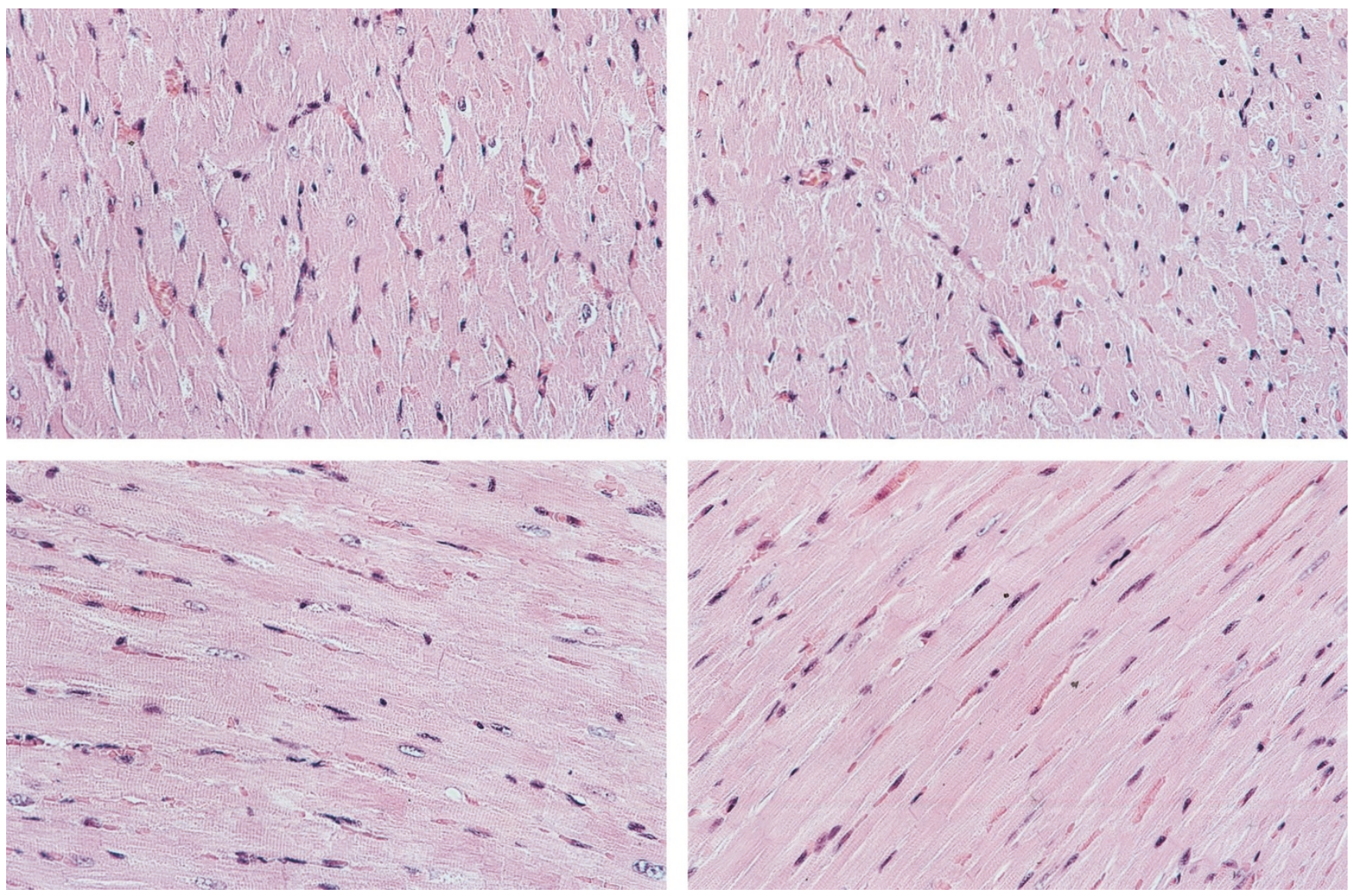

Figure 2. Hematoxylin-eosin staining, $\times 250$. Composition of SAL- (left) and DEX- (right) treated 45 -wk-old rat hearts in cross-section $($ top $)$ and in longitudinal section (bottom).

used the median values were within $2 \%$ and $8 \%$, respectively, of the median value obtained by use of a larger sample.

Statistical analysis. The data are presented as the mean \pm SD. Differences between $\mathrm{Bw}, \mathrm{Hw}, \mathrm{Hw}: \mathrm{Bw}$ ratio, DNA and Prot contents, Prot:DNA ratio, and morphometric measurements between the SAL and DEX groups at each time were assessed with $t$ test. A $p<0.05$ was considered statistically significant.

\section{RESULTS}

All groups at the various ages (d 7, 8 and 45 wk) consisted of eight rats. The animals used for the histopathologic study consisted of two additional groups of four rats each of $45 \mathrm{wk}$ of age.

Newborn rats. $\mathrm{Bw}$ and $\mathrm{Hw}$ in the DEX-treated rats were significantly lower compared with the SAL-treated rats $(p<$ 0.0001 and $p<0.0001$, respectively; Table 1). However, Hw:Bw ratio (Fig. 1, top) was higher in DEX-treated rats $(p<$ $0.05)$. Prot content tended to be higher $(p<0.09)$ and DNA content was lower $(p<0.0001)$ in DEX-treated rat pups compared with the SAL-treated rat pups. Consequently, the Prot:DNA ratio (Fig. 1, bottom) was much higher in the DEX-treated rat pups $(p<0.0001)$.

Eight-wk-old rats. The changes induced by DEX in the newborn rats were normalized in the 8-wk-old rats, that is, the differences in $\mathrm{Bw}, \mathrm{Hw}$, and $\mathrm{Hw}: \mathrm{Bw}$ ratio were not significant
(Table 1). There was a tendency for $\mathrm{Hw}$ to be lower in DEX-treated rats $(483 \pm 61 \mathrm{mg})$ compared with $\mathrm{Hw}$ in SALtreated rats $(533 \pm 59 \mathrm{mg})$. Hw:Bw ratio (Fig. 1, top), DNA content, Prot content, and the Prot:DNA ratio (Fig. 1, bottom) were not significantly different between DEX- and SAL-treated rats.

Forty-five-wk-old rats. In the adult rats the $\mathrm{Bw}$ did not differ, but $\mathrm{Hw}$ in the DEX-treated rats was significantly lower $(p<0.001)$ compared with the SAL-treated rats (Table 1). Consequently $\mathrm{Hw}: \mathrm{Bw}$ ratio (Fig. 1, top) was lower in the DEX-treated rats $(p<0.0001)$. DNA content did not differ between DEX-rats and SAL-treated rats. Prot content, however, was significantly higher in DEX-treated rats $(p<0.02)$, as was the Prot:DNA ratio (Fig. 1, bottom; $p<0.05$ ).

Histopathology. Microscopic examination of the hematoxylin-eosin-stained slides showed no striking differences between the hearts of the SAL- and DEX-treated rats. Some nuclei of myocytes in DEX-treated hearts displayed elongation and condensation of an enlarged amount of chromatin (data not shown). With this staining it is not possible to evaluate interstitial fibrosis (Fig. 2). Modified AZAN staining showed additional microscopic abnormalities. In crosssectioned myocytes of DEX-treated hearts, the diameter is increased. Myocytes were surrounded by a thin but evident layer of blue-stained collagen, indicating early degeneration of myocytes replaced by fibrous tissue (Fig. 3). In longitu- 

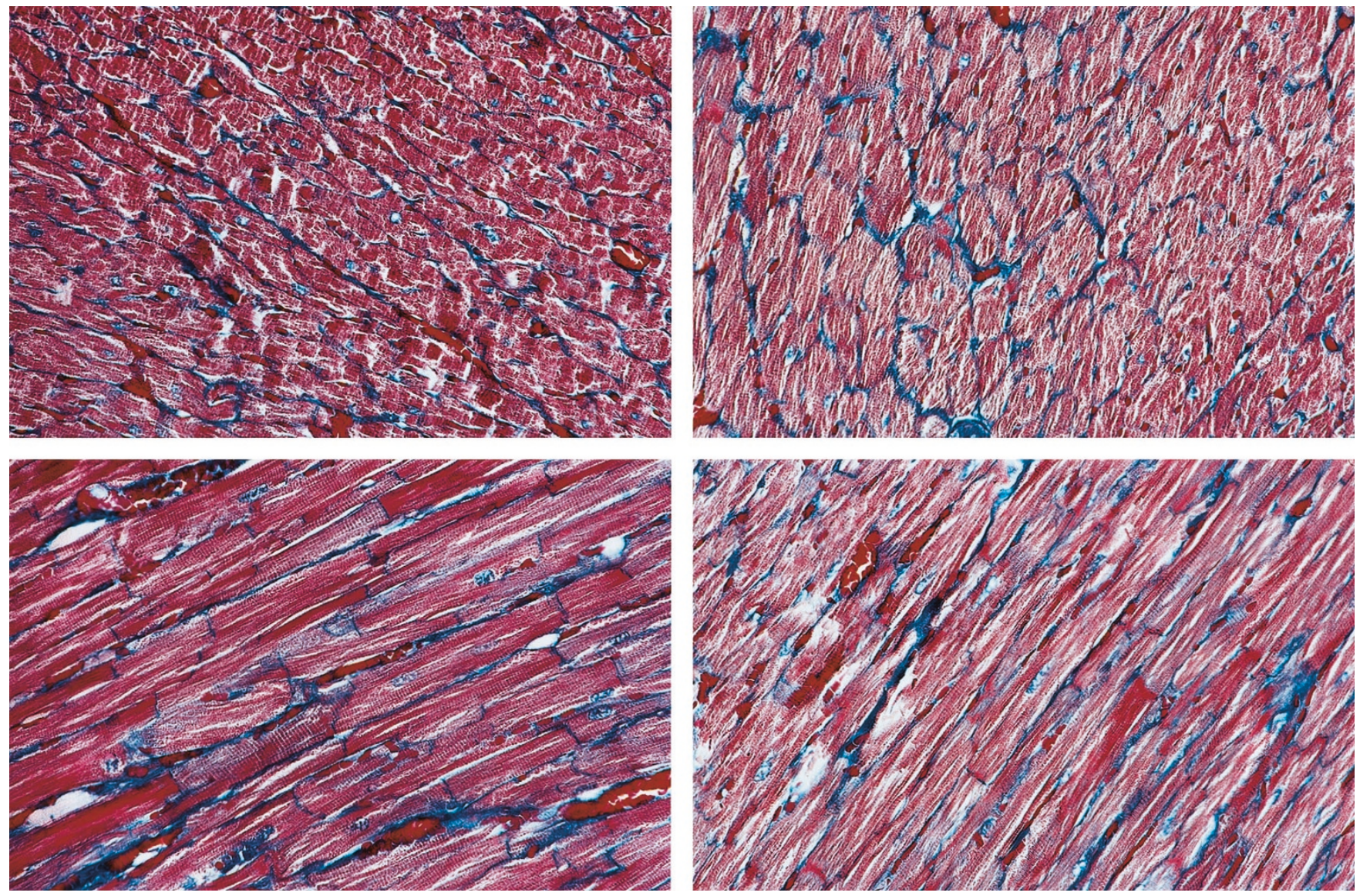

Figure 3. Modified AZAN staining, $\times 250$. Composition of SAL- (left $)$ and DEX- (right) treated 45 -wk-old rat hearts in cross-section $($ top $)$ and longitudinal section (bottom).

dinally sectioned myocytes, the distance between intercalated discs, indicating the junctions between two myocytes, was larger in the DEX-treated compared with the SALtreated rats.

Morphometry of the myocardial tissue confirmed hypertrophy in DEX-treated rats: cardiomyocyte volume was $36.3 \pm$ $2.1 \mu^{3}$ in DEX-treated rats versus $24.4 \pm 1.6 \mu \mathrm{m}^{3}$ in SAL-treated rats $(p<0.05)$. Also the length and width of cardiomyocytes of DEX-treated rats were increased: $90.1 \pm$ $4.9 \mu \mathrm{m}$ versus $73.0 \pm 0.8 \mu \mathrm{m}(p<0.05)$ and $22.7 \pm 1.2 \mu \mathrm{m}$ versus $20.7 \pm 0.6 \mu \mathrm{m}(p<0.05)$ in DEX- and SAL-treated rats, respectively. Figure 4 summarizes the results.

Collagen content was significantly higher in DEX-versus SAL-treated rats $(4.11 \pm 1.15$ vs $2.21 \pm 0.68 \% p<0.05)$.

Both microscopic findings combined with the morphometric data strongly indicate a developing hypertrophy of cardiomyocytes in DEX-treated rat hearts with signs of early fibrosis.

\section{DISCUSSION}

The present study confirms earlier studies $(9,10,15)$ of myocardial hypertrophy after perinatal and neonatal DEX treatment in the newborn animal: the increased Prot:DNA ratio, together with the lower DNA content of myocardial tissue, indicates an increase in cardiomyocyte size, but no increase in myocyte numbers in the 7-d-old DEX-treated rats. These neonatal DEX-induced changes have generally been considered to be transient, and indeed we found an apparent normalization of the measurements in the 8-wk-old animals. However, we found definite histopathologic and biochemical abnormalities in the 45 -wk-old rat myocardium, indicating that perinatal GC therapy in therapeutic doses permanently affects myocyte growth. Despite the fact that the DEX-treated rats had smaller hearts, these hearts showed definite signs of myocyte hypertrophy as indicated by the higher Prot:DNA ratio and larger myocyte volumes. Moreover, histopathologic analysis also suggests early degeneration of individual cardiomyocytes in the DEX-treated rat hearts, as indicated by the increased amount of collagen. We therefore conclude from the present study that therapeutic doses of DEX, similar to those usually administered to the newborn preterm baby eligible for GC treatment, cause permanent structural abnormalities in the myocardium.

How can these irreversible effects of neonatal GC treatment on the development of the rat heart be explained? Rudolph et al. $(10,16)$ suggested that myocyte growth is permanently inhibited after perinatal GC exposure. Normally, the fetal heart grows by cell division (hyperplasia) until term (15). After birth, the heart weight increases along with body weight by an increase in myocyte volume and size [hypertrophy (17)]. We suggest that in the very preterm infants, GC (DEX) treatment inhibits mitosis of myocytes, blocking the growth potential of the immature heart, resulting in fewer myocytes. In these 

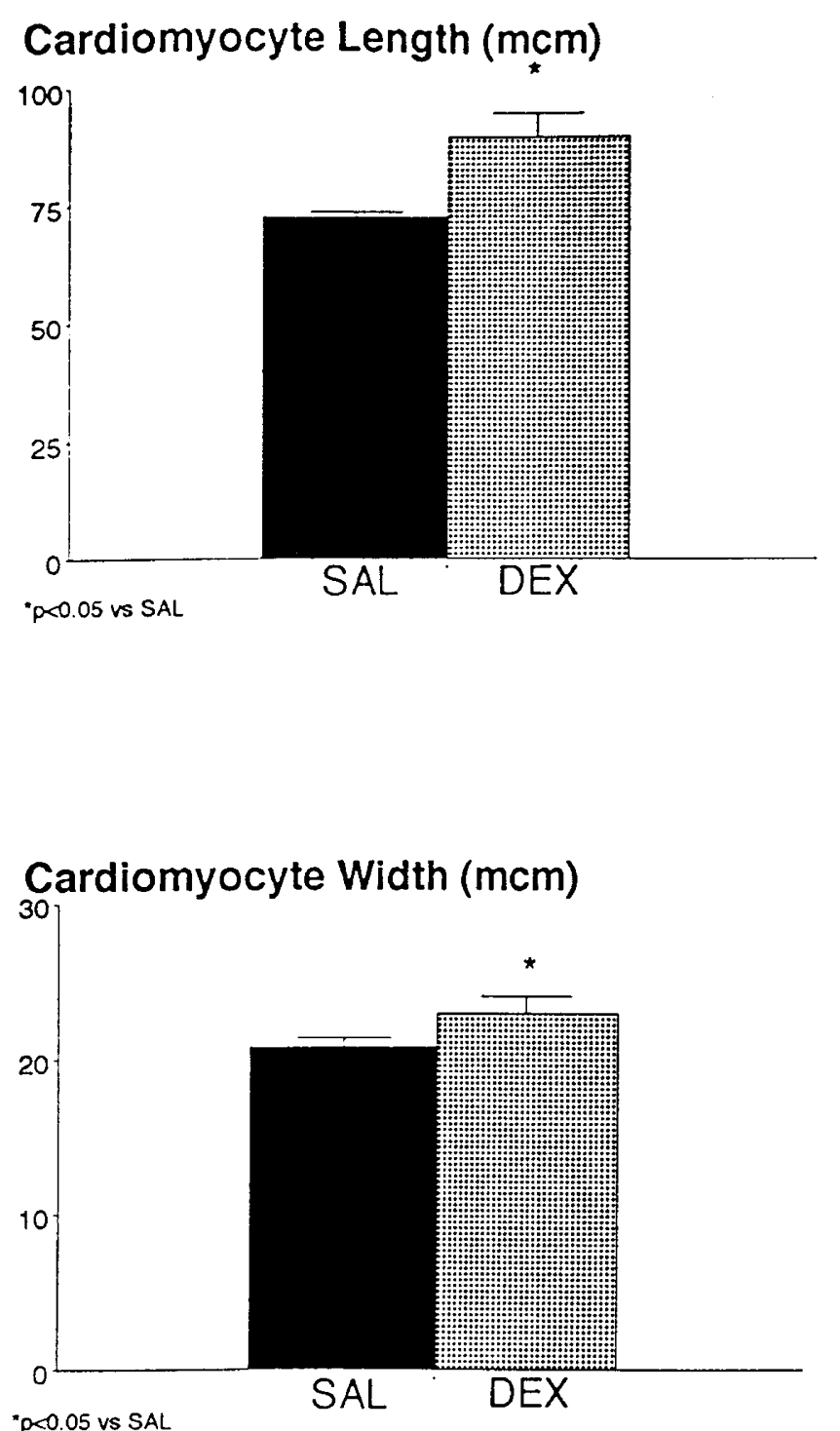

" $0<0.05$ vs SAL

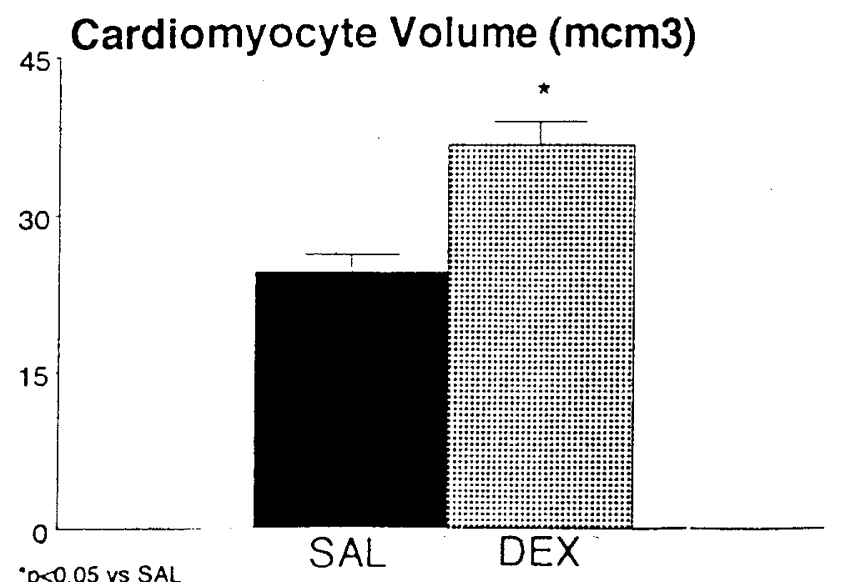

Figure 4. Mean values ( $\pm \mathrm{SD}$ ) of the length (top), width (middle), and volume (bottom) of cardiomyocytes of 45 -wk-old rats neonatally treated with DEX (strippled bars) or SAL (black bars). ${ }^{*} p<0.05$ DEX $v s$ SAL. circumstances hypertrophy will be the natural alternative for further growth of the heart to meet its hemodynamic demands. However, the premature hypertrophy may limit the reserve of the myocyte for further increase of its size (16). This appears to have occurred in the rats in the present study that were treated with DEX early in life. The low amounts of DNA in the neonatal rat hearts suggest fewer myocyte divisions compared with SAL-treated rats. These early alterations clearly require more detailed studies; however, the later effects of neonatal DEX treatment are alarming. The hearts of the 45-wk-old DEX-treated rats show typical features of compensatory remodeling of the heart (18), i.e. myocyte hypertrophy accompanied by polyploidy, as indicated by the increased size and chromatin content of the nuclei (Fig. 2). Explanations for the apparent normalization of the $\mathrm{Hw}: \mathrm{Bw}$ ratio and Prot:DNA ratio in the juvenile rats at $8 \mathrm{wk}$ of age remain speculative (i.e. delayed DNA replication and arrested cell division may result in polyploid myocytes as suggested by the DNA content in the 8 -wk-old). It is noteworthy that this observation resembles the clinical picture of normalization after cessation of therapy in the infant treated with GCs (8). At $45 \mathrm{wk}$, body weights of the DEX-treated rats are comparable to those of the SAL-treated rats, but heart weights were significantly lower, suggesting that fewer myocytes are available to meet the same hemodynamic demands compared with SAL-treated rats. The decrease in cardiac mass despite an increase in myocyte size at $45 \mathrm{wk}$ could be the result of an overall decrease in cell number as a result of reduced proliferation in the neonatal period. In addition (or alternatively) increased cardiomyocyte death or loss of cells could be involved. Thus assessment of the involvement of apoptosis or changes in proliferative activity will be needed. These analyses and several additional approaches will be carried out integratively in our laboratories in the near future.

It cannot be excluded that the hypertrophy and degenerative changes of the myocardium in the DEX-treated rats are caused by long-lasting changes in afterload of the left ventricle such as hypertension. Several studies dealing with antenatal GC treatment suggest lifelong changes in cardiovascular function $(4,5$, 19). These studies postulate that high concentrations of antenatal and perinatal GCs alter the number of steroid receptors in the hypothalamic-pituitary-adrenal axis and affect other systems such as the renin-angiotensin system. This ultimately leads to pathologic cardiovascular disorders in adults such as alterations in the autonomous nervous system leading to systemic vasoconstriction $(19,20)$. Benediktsson et al. (6) reported that GC exposure in utero leads to hypertension in adult life. Moreover, neonatal GCs also alter the epinephrine and norepinephrine content in the hypothalamus (7). However, this in utero GC-induced hypertension is less involved in the myocardial abnormalities reported in our study, because it does not explain the lower $\mathrm{Hw}$ and amount of DNA. It may, however, add to the histopathologic abnormalities detected in the hearts of DEX-treated rats: These myocytes are already limited in their growth responsiveness and may suffer irreversible changes when a second strain occurs, such as increase in left ventricular afterload. The tendency of a lower Hw at $8 \mathrm{wk}$ and the definitely lower $\mathrm{Hw}$ at 45 wk of age after DEX treatment in the neonatal period, together with the signs of 
hypertrophy and of precocious aging, make it probable that during adult life these hearts reach their limits in their ability to respond to requirements of increased growth. Indeed, preliminary results suggest that the life expectancy of rats that have undergone similar DEX treatments to those used in the present study is significantly reduced (21). An important issue relates to the development of fibrosis. There is a considerable literature on the development of fibrosis in the adult heart with left ventricular hypertrophy $(22,23)$, and it has been suggested (24) that the fibrosis develops as a result of the myocyte hypertrophy outgrowing the development of new capillaries, so that there is a reduction of oxygen supply to the myocardium. In our studies, we do not have information about vascular supply, but it is quite possible that DEX has also affected the growth of the coronary circulation, so that it has a limited ability to increase with myocyte hypertrophy.

We do not know whether there are species differences with respect to the vulnerability for neonatal DEX treatment of the adult rat heart compared with the human heart. Although it is postulated that the neonatal rat is more prone to GC-induced negative long-term effects, this is only substantiated for the immune system (25). Moreover, mostly pharmacologic dosages of DEX are used in these animals, which are commonly much higher compared with doses used in clinical practice in the preterm infant. On the other hand, data of eight recent randomized-controlled trials were entered into a meta-analysis to determine the long-term effects of neonatal GC therapy. This study showed increased rates of cerebral palsy and impaired neurodevelopmental outcome (26).

Nonetheless, until it is proven that neonatal DEX treatment has no negative effects on the human adult heart (unlike the situation in our rat model), the use of GC therapy in premature infants raises serious ethical concerns. Clinical studies are not possible yet because neonatally treated humans are too young for complete long-term studies of the cardiovascular system. However, hemodynamic, histopathologic, and moleculargenetic characterization of the developing and adult heart in relation to perinatal GC treatment are of utmost importance for early detection and possible treatment of heart failure in the large number of individuals already treated for prevention of chronic lung disease and often in rather high dosages as was common in the early 1990s. Therefore, one should be aware of the worrying results of this and other studies related to perinatal GC treatment.

\section{CONCLUSIONS}

In conclusion, neonatal DEX treatment in rat pups causes a permanent decrease in Hw, probably caused by inhibition of myocyte mitotic activity in this period. In the adult rat heart this treatment leads to hypertrophy and precocious degeneration of cardiomyocytes. These findings may become of utmost importance for a potentially large patient population.

Irrespective of the present study, which was not designed for this purpose, it remains important to investigate whether treat- ment with lower dosages and shorter duration of GCs are still effective to prevent or reduce neonatal chronic lung disease without long-term side effects on the myocardium.

Acknowledgments. The authors thank Prof. J.R.G. Kuipers for his stimulating support, J. Takens for technical assistance, and Profs. A.M. Rudolph and P.J.J. Sauer for critically reading the manuscript and making helpful suggestions.

\section{REFERENCES}

1. Mammel HC, Johnson DE, Green TP, Thomson TR 1983 Controlled trial of dexamethasone therapy in infants with bronchopulmonary dysplasia. Lancet 1:1356-1358

2. Cummings JJ, D’Eugenio DB, Gross SJ 1989 a controlled trial of dexamethasone in preterm infants at high risk for bronchopulmonary dysplasia. N Engl J Med 320:1505-1510

3. Felszeghy K, Gaspar E, Nyakas C 1996 Long-term selective down-regulation of brain glucocorticoid receptors after neonatal dexamethasone treatment in rats. J Neuroendocrinol 8:493-499

4. Levitt NS, Lindsay RS, Holmes MC, Seck1 JR 1996 Dexamethasone in the last week of pregnancy attenuates hippocampal glucocorticoid receptor gene expression and elevates blood pressure in the adult offspring in the rat. Neuroendocrinology 64:412418

5. Dodic M, May CN, Wintour EM, Coghlan JP 1998 An early prenatal exposure to excess glucocorticoid leads to hypertensive offspring in sheep. Clin Sci 94:149-155

6. Benediktsson R, Lindsay RS, Noble J, Seckl JR, Edwards CRW 1993 Glucocorticoid exposure in utero: new model for adult hypertension. Lancet 341:339-341

7. De Kloet ER, Rosenfeld P, van Eekelen JA, Sutanto W, Levine S 1988 Stress glucocorticoids and development of the brain. Prog Brain Res 73:101-120

8. Werner JC, Sicard RE, Hansen WR, Soloman E, Cowett RM, Oh W 1992 Hypertrophic cardiomyopathy associated with dexamethasone therapy for chronic lung disease. J Pediatr 120:286-291

9. La Maer NS, MacGilvray SS, Myers TF 1997 Dexamethasone-induced myocardial hypertrophy in neonatal rats. Biol Neonate 72:175-180

10. Rudolph AM, Roman C, Gournay V 1999 Perinatal myocardial DNA and protein changes in the lamb: effect of cortisol in the fetus. Pediatr Res 46:141-146

11. Knowlton K, Mancini M, Creason S, Morales C, Hockenbery D, Anderson BO 1998 Bcl-2 slows in vitro breast cancer growth despite its antiapoptotic effect. J Surg Res $76: 22-26$

12. Smith PK, Krohn RI, Hermanson GT, Mallia AK, Gartner FH, Provenzano MD, Fujimoto EK, Goeke NM, Olson BJ, Klenk DC 1985 Measurement of protein using bicinchoninic acid. Anal Biochem 150:76-85

13. Vliegen HW, van der Laarse A, Huysman JAN, Wijnvoord EC, Mentar M, Cornelisse CJ, Eulderingk F 1987 Morphometric quantification of myocyte dimensions validated in normal growing rat hearts and applied to hypertrophic human hearts. Cardiovasc Res 21:352-357

14. Van Oosterhout MF, Prinzen FW, Arts T, Schreuder JJ, Vanagt WY, Cleutjens JP, Reneman RS 1998 Asynchronous electrical activation induced asymmetrical hypertrophy of the left ventricular wall. Circulation 98:588-595

15. Sicard RE, Werner JC 1992 Dexamethasone induces a transient relative cardiomegaly in neonatal rats. Pediatr Res 31:359-363

16. Rudolph AM 2000 Myocardial growth before and after birth: clinical implications. Acta Paediatr 89:129-133

17. Smolich JJ, Walker AM, Campbell GR, Adamson TM 1989 Left and right ventricular morphometry in fetal, neonatal, and adult sheep. Am J Physiol 257:H1-H9

18. Herget GW, Neuburger M, Plagwitz R, Adler CP 1997 DNA content, ploidy level and number of nuclei in the human heart after myocardial infarction. Cardiovasc Res $36: 45-51$

19. Dodic M, Peers A, Coghlan JP, Wintour M 1999 Can excess glucocorticoid, in utero, predispose to cardiovascular and metabolic disease in middle age? Trends Endocrinol Metab 10:86-91

20. Barker DJP 1997 Fetal nutrition and cardiovascular disease in later life. Br Med Bull 53:96-108

21. Kamphuis PJGH, Croiset G, Bakker JM, Wiegant VM, Van Bel F 2001 Neonatal dexamethasone treatment results in a reduction of life-span in rats. Pediatr Res 49:299A(abstr)

22. Eghbali M, Weber KT 1990 Collagen and the myocardium: fibrillar structure, biosynthesis and degradation in relation to hypertrophy and its regression. Mol Cell Biochem 96:1-14

23. Zhu YC, Zhu YZ, Spitznagel H, Gohlke P, Unger T 1996 Substrate metabolism, hormone interaction, and angiotensin-converting enzyme inhibitors in left ventricular hypertrophy. Diabetes 45(suppl 1):S59-S65

24. Brixius B, Lindinger A, Baghai A, Limbach HG, Hoffmann W 1999 Ventricular tachycardia after erythromycin administration in a newborn with congenital AVblock. Klin Paediatr 211:465-468

25. Claman HN 1972 Corticosteroids and lymphoid cells. N Engl J Med 287:388-397

26. Barrington KJ 2001 The adverse neuro-developmental effects of postnatal steroids in the preterm infant: a systematic review of RCTs. BMC Pediatr 1:1-8 